Check for updates

Cite this: RSC Adv., 2018, 8, 27253

Received 19th May 2018

Accepted 2nd July 2018

DOI: $10.1039 / c 8 r a 04273 j$

rsc.li/rsc-advances

\section{Bio-inspired microcapsule for targeted antithrombotic drug delivery $\dagger$}

\author{
Wei Ye, (D) ${ }^{a}$ Nan Wang, ${ }^{a}$ Kebang Hu, (D) *b Lincai Zhang, ${ }^{a}$ Aihui Liu, (D) a \\ Changjiang Pan, (D)*a Tao Gong, ${ }^{a}$ Tao Liu ${ }^{a}$ and Hongyan Ding ${ }^{a}$
}

Thrombosis or embolism is the leading cause of death and long-term adult disability worldwide. To reduce the risk of thrombosis and hemorrhaging in patients, a facile and versatile method was developed to fabricate microcapsules for targeted antithrombotic drug delivery. The microcapsules were prepared via oxidative polymerization of dopamine on polystyrene microspheres, followed by immobilization of fibrinogen onto the surface of poly(dopamine) layers. Subsequently, microcapsules were obtained by removing the cores with THF. Nattokinase was loaded into the microcapsules via diffusion. The loading amount was approximately $0.05 \mathrm{mg} \mathrm{g}^{-1}$ at $37{ }^{\circ} \mathrm{C}$, and the loading efficiency was nearly $75 \%$, based on the initial concentration of nattokinase in PBS. The release of nattokinase was a gradual process at $37^{\circ} \mathrm{C}$, and the activity of the targeted activated platelets was highly efficient. The antithrombotic activity of the nattokinase microcapsules was evidenced by the sharp dissolution of fibrin clots and the blood clotting time indexes. A gradual release mechanism of platelet-inspired microcapsules used for targeted antithrombotic therapy was proposed. This strategy for targeted antithrombotic drug delivery, which lowers the demand dose and minimizes side effects while maximizing drug efficacy, provides a potential new way to treat life-threatening diseases caused by vascular disruption.

\section{Introduction}

The obstruction of blood flow to the heart, lungs and brain is the leading cause of death and long-term adult disability worldwide. ${ }^{1,2}$ This disease-causing arterial obstruction is caused by blood platelets, which activate, adhere to the vascular wall, and then form a fibrin meshwork, finally perpetuating and stabilizing as a platelet plug., ${ }^{3,4}$ The balance between the formation of fibrins from fibrinogen during proteolysis by thrombin and hydrolysis of fibrin clots by plasmin plays a significant role in preventing thrombosis. ${ }^{5}$ Plasminogen activator and plasmin-like proteins are the two kinds of antithrombotic drugs used to decrease the risk of thrombosis. ${ }^{6}$ Platelet inhibitors, such as aspirin and clopidogrel, can be used to reduce thrombosis as well. ${ }^{7}$ However, an undesirable increase in bleeding is produced when these agents are administered at their most efficient antithrombotic doses. ${ }^{8}$ Thus, it is urgent to design a thrombolytic delivery system that can administer drugs to target thrombi and increase the local drug concentration, without affecting platelet function. Nattokinase (NK) is a fibrinolytic enzyme ${ }^{9}$ that acts directly to degrade

ajiangsu Provincial Key Lab for Interventional Medical Devices, Huaiyin Institute of Technology,Huaian 223003, China. E-mail: panchangjiang@hyit.edu.cn

${ }^{b}$ Department of Urology, The First Hospital of Jilin University, Changchun 130021, PR China. E-mail: hukebang1980@126.com

$\dagger$ Electronic supplementary information (ESI) available. See DOI: 10.1039/c8ra04273j the fibrin in blood clots via hydrolysis or its ability to convert plasminogen to plasmin, reducing the thrombi faster and without increasing the risk of bleeding. ${ }^{10}$ Therefore, loading NK into a controlled delivery system is a promising approach to achieve targeted antithrombosis.

Pathologic thrombus formation in the arterial system occurs when diseases lead to unwarranted platelet activation, resulting in a local aggregation of platelets, as well as other blood cells and plasma proteins. ${ }^{\mathbf{1 1 , 1 2}}$ During platelet activation, large quantities of glycoproteins (GPs), such as GPIIb/IIIa, GP IX, and GP V, are expressed on the surfaces of platelets. Theses glycoproteins can bind most of the agonists, including von Willebrand factor (vWF), collagen and fibrinogen (Fib), which render platelets highly adhesive by attaching to vessel walls and stabilizing platelet plugs through cohesive interactions between platelets. $^{13}$ Inspired by the adhesion of activated platelets, synthetic particles ${ }^{\mathbf{1 4}-\mathbf{1 6}}$ have been fabricated to mimic the shape, size and elasticity of natural platelets, and thus investigate migration and wall interactions. ${ }^{17,18}$ However, it is still a challenge to integrate the physical-mechanical properties and biological functions of platelets into platelet-mimetic particles for targeted antithrombotic drug delivery.

Polymer microparticles with well-defined structures and adaptable properties have potential applications in drug loading and controlled release, because they often possess a structurally intact hollow capsule achieved by dissolution of the template. ${ }^{19}$ Polydopamine (PDA), formed by oxidation of 
dopamine, is composed of dihydroxyindole, indoledione, and dopamine units. ${ }^{20}$ It is easy to create an adhesive coating on a variety of materials through the spontaneous oxidative polymerization of dopamine, ${ }^{21}$ to provide a versatile, functional platform for secondary reactions. ${ }^{22}$ By combining polymer microparticles and a PDA coating, a drug delivery carrier with biocompatibility and antioxidant activity can be prepared, in which NK can be loaded as an antithrombosis agent. ${ }^{23,24}$

Previously, we fabricated PDA-coated micro/nano-particles and investigated the platelet-particle surface interactions. ${ }^{25}$ Here, our continuing work was extended to modify the PDA@PS coating, to subsequently obtain microcapsules by removing their cores with THF. The size and the morphology of the microparticles were assessed using SEM. NK loading and release were monitored via high-performance liquid chromatography. Finally, the NK-loaded microcapsules were used to target platelets alone and as an antithrombosis therapy in a complex blood environment.

\section{Materials and methods}

\subsection{Chemicals and materials}

Monodisperse PS particles ( $3 \mu \mathrm{m}$ diameter) were purchased from Suzhou Nano-micro Technology Company (China). Dopamine, Fib and thrombin were purchased from Sigma-Aldrich. Nattokinase (NK, $410 \mathrm{IU} \mathrm{g}^{-1}$ ) was obtained from Wako (Japan). Tris(hydroxymethyl)aminomethane (Tris, $\mathrm{pH}$ 8.5) was purchased from Shanghai Dingguo Biotechnology Company (China). Tetrahydrofuran (THF, analytical grade) was obtained from Shanghai Chemical Reagent Co., Ltd., and used without further purification. Phosphate-buffered saline (PBS 0.01 M phosphate buffer, $\mathrm{pH}$ 7.4), used for NK loading, fibrinolysis, and blood clotting experiments, was freshly prepared. Other reagents were AR grade and used without further purification.

\subsection{Preparation of PDA-Fib microcapsules}

The PDA coating on the surface of the PS microspheres was formed through the in situ spontaneous oxidative polymerization of dopamine. $10 \mu \mathrm{L}$ of PS microspheres $\left(100 \mu \mathrm{g} \mathrm{mL}{ }^{-1}\right)$ was added to Tris/HCl (10 mM, pH 8.5) buffer with $0.15 \mathrm{mg} \mathrm{mL}^{-1}$ dopamine to prepare PDA-coated PS particles (PS@PDA). The mixed solution was stirred for a predefined period at $37^{\circ} \mathrm{C}$, and then centrifuged at $3000 \mathrm{rpm}$, followed by washing with Tris/ $\mathrm{HCl}$ buffer until the supernatant became colorless. The obtained PS@PDA particles were added into PBS solution with $0.5 \mathrm{mg} \mathrm{mL}{ }^{-1}$ Fib and stirred for $6 \mathrm{~h}$ at room temperature. After washing with distilled water and freeze-drying, Fib-bonded microparticles (PS@PDA-Fib) were formed.

The uncrosslinked PS microspheres could be easily etched by THF solution. First, $1 \mathrm{mg}$ of PS@PDA-Fib microparticles was added to $4 \mathrm{~mL}$ of THF solution. PDA-Fib microcapsules were obtained by etching the PS cores from the PS@PDA-Fib microparticles with $\mathrm{THF}^{.6}$ After stirring at room temperature for $24 \mathrm{~h}$, the solution was centrifuged at $3000 \mathrm{rpm}$ and washed with water and ethanol at least three times to remove the residual solvent. Lastly, the microparticles were dried in a vacuum oven overnight at $30^{\circ} \mathrm{C}$.

\subsection{NK loading and delivery}

NK was loaded into PDA-Fib microcapsules via concentrationcontrolled diffusion. PDA-Fib microcapsules (1 $\mathrm{mg}$ ) were added to $5 \mathrm{~mL}$ of PBS solution containing $1 \mathrm{wt} \%$ bovine serum albumin (BSA), $0.05 \mathrm{wt} \%$ Tween 20 , and $1 \mathrm{mg} \mathrm{mL}^{-1} \mathrm{NK} .{ }^{27}$ BSA was used as a blocking agent to prevent non-specific binding when PDA-Fib microcapsules interacted with NK and to maintain the activity of NK. The combined use of Tween 20 and BSA provided the best conditions to decrease most of the nonspecific binding. The mixture was placed on a shaking table overnight at $20{ }^{\circ} \mathrm{C}$. Then, the solution was centrifuged at $3000 \mathrm{rpm}$ and washed with deionized water at least three times. After drying in a vacuum oven overnight at $30{ }^{\circ} \mathrm{C}$, NK-loaded PDA-Fib microcapsules were obtained.

NK-loaded PDA-Fib microparticles were incubated in PBS solution at room temperature. Then, $1 \mathrm{~mL}$ of particle solution was collected at $10 \mathrm{~min}$ to $3 \mathrm{~h}$, and the release of NK was monitored via high-performance liquid chromatography (Waters 600 HPLC, evaporative light scattering detector) using a standard calibration curve. Acetonitrile was used as the mobile phase with the injection volume of $10 \mu \mathrm{L}$, and the run time was $10 \mathrm{~min}$ at the absorbance wavelength of $230 \mathrm{~nm} .{ }^{28}$ The total loading concentration was assumed to be the equilibrium concentration of released NK.

\subsection{Targeting activated platelets}

Fresh blood collected from a healthy rabbit was immediately mixed with $3.8 \mathrm{wt} \%$ sodium citrate solution at a dilution ratio of $9: 1$. This study was performed in strict accordance with the NIH guidelines for the care and use of laboratory animals (NIH Publication no. 85-23 Rev. 1985) and was approved by the Ethical Committee of the Chinese Academy of Sciences (Beijing, China). Platelet rich plasma (PRP) was obtained from the supernatant after centrifugation of whole blood at $1000 \mathrm{rpm}$ for 15 min. PS, PS@PDA, PS@PDA-Fib and NK microcapsules were incubated with platelets at $37{ }^{\circ} \mathrm{C}$ for $30 \mathrm{~min}$ with stirring to assess the targeting properties of Fib-bonded PDA. This was followed by deposition of the particles on polymer substrates for SEM analysis (XL 30 ESEM FEG, FEI Company).

\subsection{Fibrinolysis activity assay}

Fib was dissolved in PBS ( $\mathrm{pH} 7.4$ ) at a concentration of $3 \mathrm{mg}$ $\mathrm{mL}^{-1}$. Then, $100 \mu \mathrm{L}$ of Fib solution was transferred into 96-well plates, followed by the addition of $20 \mu \mathrm{L}$ of thrombin $\left(5 \mathrm{U} \mathrm{mL}^{-1}\right)$ to form a fibrin clotting. Next, $100 \mu \mathrm{L}$ samples of a PBS suspension of PS@PDA-Fib $\left(0.5 \mathrm{mg} \mathrm{mL}^{-1}\right)$ and a PBS suspension of NK-loaded microcapsules $\left(0.5 \mathrm{mg} \mathrm{mL}^{-1}\right)$ were added separately to 96-well plates, which were immediately placed in a microplate reader. The absorbance measurements at $630 \mathrm{~nm}$ were recorded every $1 \mathrm{~min}$ for $130 \mathrm{~min}$. Meanwhile, $200 \mu \mathrm{L}$ of PBS was added to 96-well plates and analyzed as a blank control.

\subsection{Whole blood clotting time}

The anticoagulant properties of PDA-Fib and NK-loaded microcapsules were evaluated using fresh rabbit blood, by the 
clotting time method. Briefly, $15 \mu \mathrm{L}$ of fresh blood and $15 \mu \mathrm{L}$ of PBS suspension with microcapsules $\left(0.5 \mathrm{mg} \mathrm{mL}^{-1}\right)$ were mixed and dropped onto the polymer substrates, followed by incubation at $37^{\circ} \mathrm{C}$ for a given time. Then, $3 \mathrm{~mL}$ of deionized water was added to stop the reaction. The concentration of free hemoglobin in water was measured using a TECAN absorbance reader (TECAN GENIOS, Austria) at $540 \mathrm{~nm}$. The relative absorbance of $30 \mu \mathrm{L}$ of whole blood diluted by $3 \mathrm{~mL}$ distilled water was assumed to be 100 . The blood clotting index (BCI) of a biomaterial can be quantified using the following equation: ${ }^{29}$

$$
\mathrm{BCI}(\%)=\frac{A_{\mathrm{s}}}{A_{0}} \times 100 \%
$$

where $A_{\mathrm{s}}$ and $A_{0}$ represent the absorbance of the sample and fresh blood, respectively.

\section{Results and discussion}

\subsection{Fabrication and characterization of microcapsules}

Platelet-inspired particles (PDA-Fib) were synthesized via the oxidative polymerization of dopamine on PS microspheres, after which Fib molecules were immobilized on the surface of the PDA layer, followed by etching of the PS core with THF (Fig. 1). The obtained microcapsules were used to load and release NK for antithrombotic activity by diffusion. ${ }^{30}$ Fib molecules immobilized on the surface of PDA have a high affinity to platelet membrane glycoproteins, such as GP IIb/IIIa and GP V, which play a crucial role in hemostasis. Thus, combined with the specific recognition of Fib and the fibrinolysis of NK, NKloaded microcapsules can target activated platelets and deliver NK to the site of thrombi for antithrombotic therapy.

The monodisperse PS microspheres of $3 \mu \mathrm{m}$ diameter were used as an initial template. Dopamine was spontaneously polymerized and oxidized into quinine and rearranged into 5,6dihydroxyindole. It then polymerized to form a PDA coating on the PS microspheres. ${ }^{31}$ Next, Fib molecules were conjugated onto the PDA-coated microspheres via the reaction of amino groups on the surface of PDA and carboxyl groups on Fib. The structures and compositions of the samples have been reported in our previous work, ${ }^{25}$ and bovine serum albumin and Fib were grafted onto the surfaces of PS@PDA particles for interacting with blood platelets.

The samples were further deposited onto a thermoplastic elastomer substrate to analyze the morphologies of the microparticles and microcapsules before and after PS etching. SEM images of PS, PS@PDA and PS@PDA-Fib microspheres and a PDA-Fib microcapsule are shown in Fig. 2. The PS microspheres with a diameter of $3 \mu \mathrm{m}$ have clear and smooth borders. After PDA and Fib modification, the surfaces of the PA@PDA microspheres are rough, due to the polymerization of PDA, and some tiny bumps are apparent on the surfaces of the PS@PDAFib microspheres. The size of the PS@PDA microspheres is approximately $3.4 \mu \mathrm{m}$ (Fig. S1 $\dagger$ ), which is similar to that of natural platelets. Significantly, after the PS core was etched, the size of particles became larger, the size distribution widened (Fig. S2 $\dagger$ ), and the microspheres collapsed into microcapsule structures (Fig. 2d). The size of the microcapsules is larger than $4 \mu \mathrm{m}$ (Fig. S2 $\dagger$ ). This hollow microcapsule is an excellent carrier to load drugs or other functional molecules and release them at target sites. ${ }^{19}$ When the etching time was $12 \mathrm{~h}$, the PS core was removed entirely (Fig. S1: $\dagger$ fourth line); when the etching time was just $3 \mathrm{~h}$, the PS core was etched partly (Fig. S1: $\dagger$ third line). Some crushed microcapsules in Fig. S1† proved that the PS core was gradually removed and the inner wall was full of coarse nicks. In the next loading and release system, fully etched microparticles were used to load NK for targeted therapy.

\subsection{NK loading and release}

NK is a thrombolytic enzyme that possesses plasminogen activation activity and directly digests fibrin through limited proteolysis. The fibrinolysis activity is retained in blood for more than 3 h. ${ }^{32}$ The encapsulation of NK into PDA-Fib microcapsules was accomplished by mixing dry PDA-Fib microcapsules in a PBS solution containing BSA, Tween 20 and NK for $12 \mathrm{~h}$ at room temperature. After equilibrium encapsulation, the NK-loaded microcapsules were obtained by centrifugation. Fig. 3 shows the release of NK from the microcapsules in PBS at

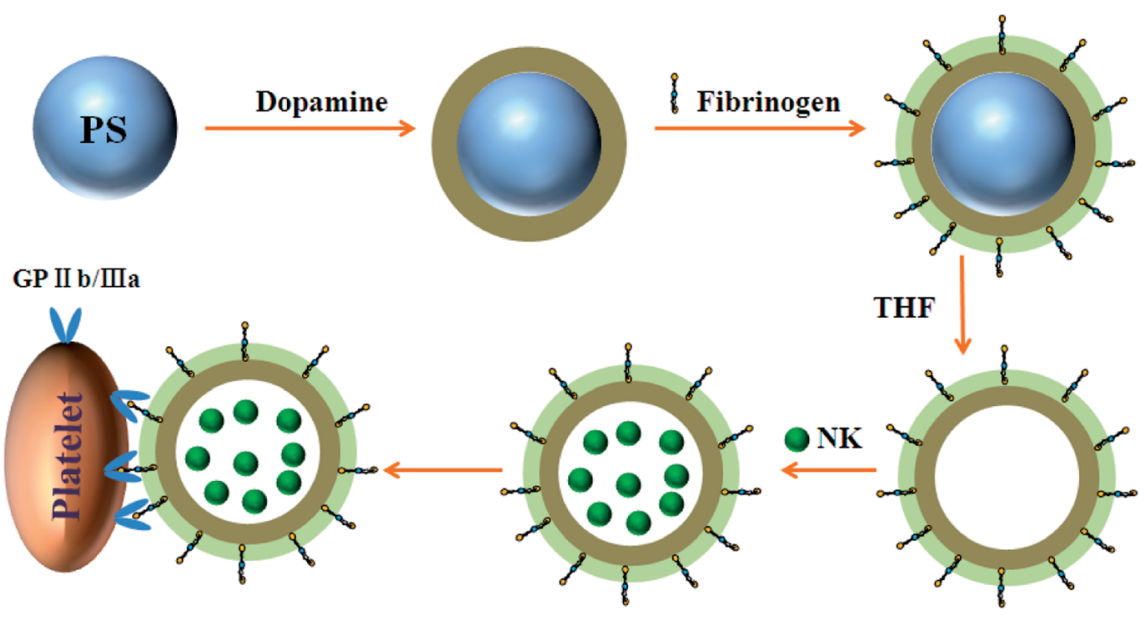

Fig. 1 Schematic illustration of the preparation of NK-loaded PDA particles and targeted drug delivery to the site of platelet plugs. 

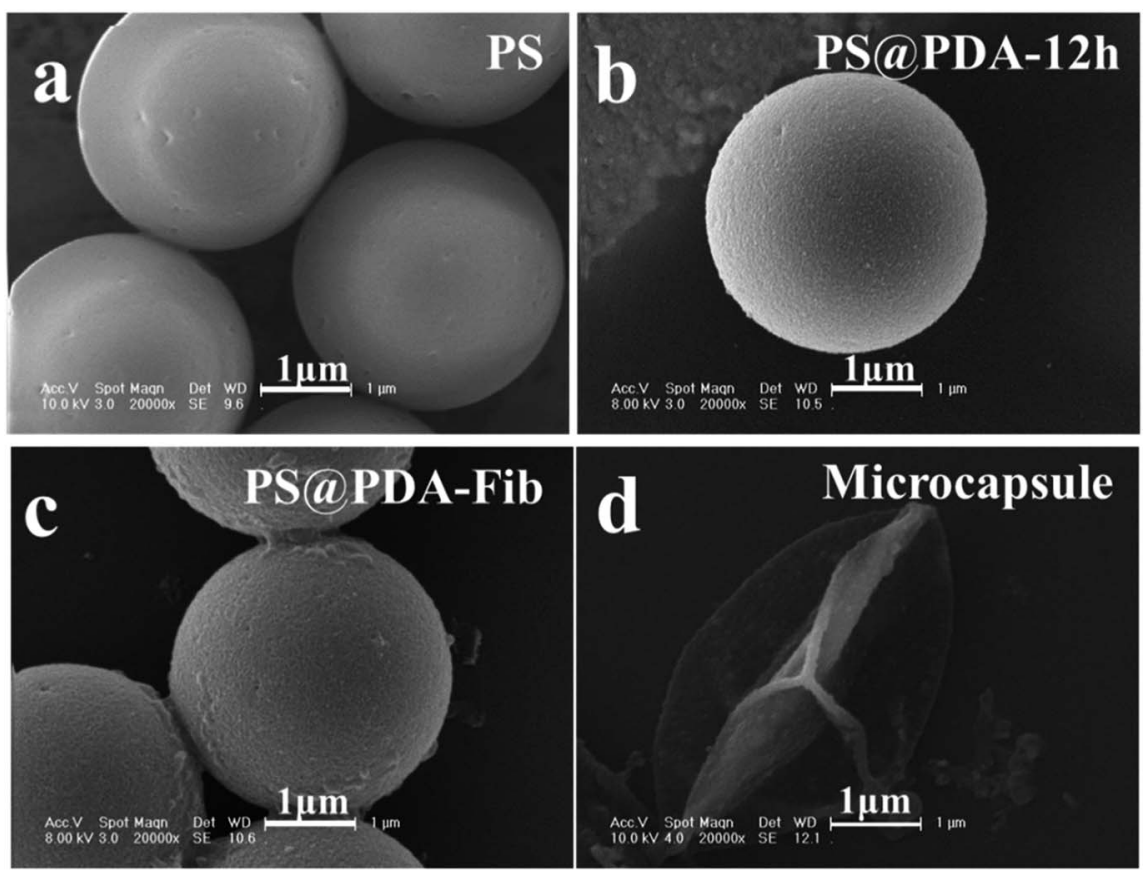

Fig. 2 SEM images of (a) PS microspheres; (b) PS@PDA microparticles; (c) PS@PDA-Fib microparticles; and (d) PDA microcapsule.

$25{ }^{\circ} \mathrm{C}$ and $37{ }^{\circ} \mathrm{C}$. It is evident that the release of $\mathrm{NK}$ at $37{ }^{\circ} \mathrm{C}$ is slightly quicker than that at $25^{\circ} \mathrm{C}$ and the final released amount is larger as well. The cumulative release of $\mathrm{NK}$ at $37^{\circ} \mathrm{C}$ is almost $45 \%$ in $30 \mathrm{~min}$. After that, the speed of release slows down to a gradual release. The slow release of $\mathrm{NK}$ at $37^{\circ} \mathrm{C}$ is similar to what would be observed under physiological conditions, especially in the case of thrombolysis in blood. Moreover, the release is limited by the diffusion of drug through the matrix structure. Assuming equilibrium release is equivalent to the loading amount of $\mathrm{NK}$, the loading amount of $\mathrm{NK}$ is approximately $0.05 \mathrm{mg} \mathrm{g}^{-1}$ at $37^{\circ} \mathrm{C}$, and the loading efficiency is nearly $75 \%$ based on the initial concentration of NK in PBS. Thus, the loaded NK microcapsules, with high loading and release efficiency at the site of thrombi, can be used for slow drug release and have potential for thrombolysis in blood.

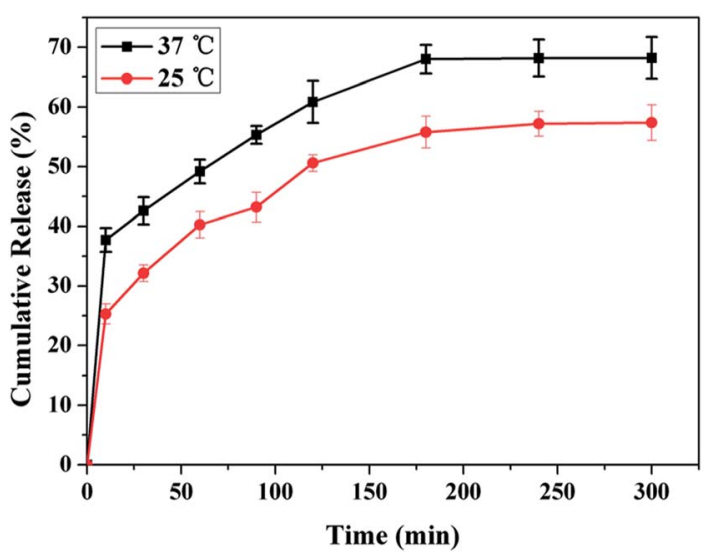

Fig. 3 Release profile of NK from PDA-Fib microcapsules (errors: mean $\pm \mathrm{SD} ; n=3$ ).

\subsection{Targeting activated platelets}

Targeting activated platelets to release antithrombotic drugs plays a key role in the treatment of thrombosis. ${ }^{33}$ Platelets are activated and form pseudopodium when the coagulation pathway is initiated. NK-loaded PDA-Fib microcapsules are excellent carriers to target activated platelets in blood by interacting with Fib and then dissolving a thrombus by gradually releasing NK molecules. PS@PDA-Fib and NK-loaded microcapsules were incubated with platelet rich plasma solution at $37^{\circ} \mathrm{C}$ for $30 \mathrm{~min}$ under stirring to target activated platelets. They were then deposited on polymer substrates to check their targeting properties. For comparison, the targeting ability of PS and PS@PDA particles was determined at the same time. Fig. 4 shows SEM images of microparticles interacting with platelets and free NK interacting with platelets as a reference. During stirring, platelets were activated. The adhesion and spreading of platelets were observed on the surface of PS microspheres, and nonspecific interactions led to PS aggregation (Fig. 4a and $\mathrm{f}$ ). After PDA modification, no platelets adhered on the surfaces of the PS@PDA particles (Fig. 4g) because of the excellent hemocompatibility of PDA. Here, we also found that the platelets and PS@PDA particles were discrete and performed almost no targeting. In contrast, PS@PDA-Fib showed high targeting ability to recognize activated platelets (Fig. 4h). Several platelets adhered or spread on one PS@PDA-Fib microparticle, which can maximize drug efficiency during thrombolysis when platelets adhere and aggregate in blood. The affinity of the plateletinspired microparticles is mainly due to the specific interactions between Fib and glycoproteins on the surfaces of activated platelets. $^{34}$ Compared with PS microparticles, PS@PDA-Fib microparticles have the advantage of acting as a drug carrier with high targeting ability, rather than inducing aggregation 


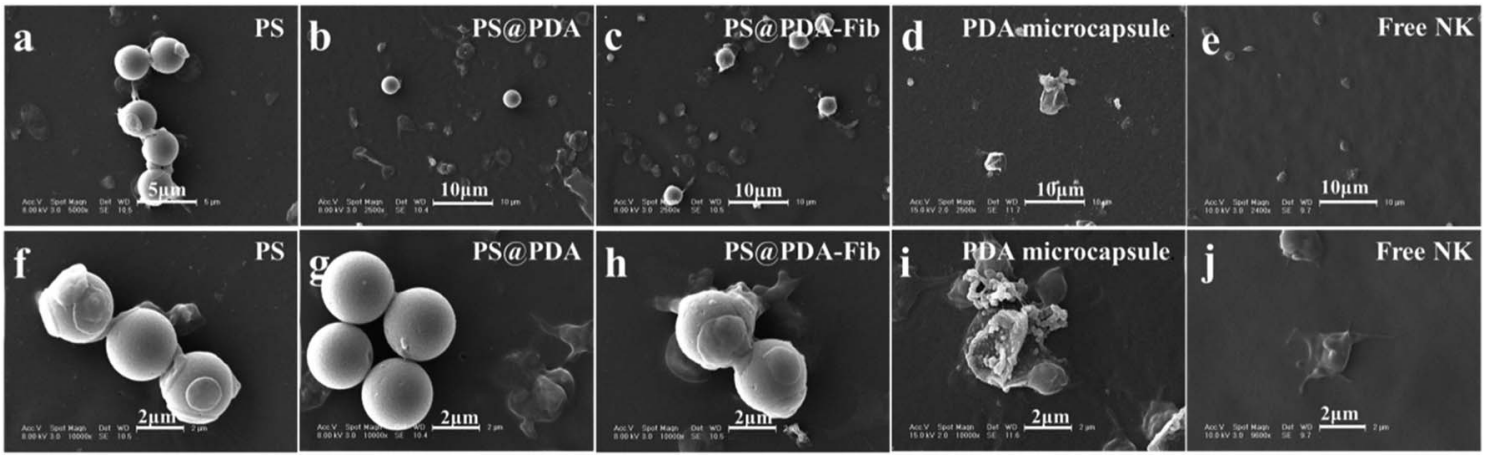

Fig. 4 SEM images of microparticles interacting with platelet rich plasma: (a and f) PS microspheres; (b and g) PS@PDA microspheres; (c and h) PS(APDA-Fib microspheres; (d and i) NK microcapsule; (e and j) free NK.

and recognition between microparticles and platelets. Significantly, when the NK-loaded microcapsules interacted with platelets, few platelets were found in the system (Fig. 4d). This suggests that the released NK can dissolve activated platelets, thus preventing a thrombus from forming. Moreover, the number of adhered platelets (described in the ESI $\dagger$ ) further confirms the thrombolysis activity of the NK-loaded microcapsules. Therefore, the modification of PDA endows the microparticles with blood compatibility, and Fib molecules can target with high efficiency. Furthermore, the release of NK from the microcapsules leads to remarkable thrombolysis activity.

\subsection{Antithrombotic activity of NK microcapsules}

To evaluate the antithrombotic activity of the NK-loaded PDA microcapsules, $100 \mu \mathrm{L}$ of a suspension of the platelet-inspired microcapsules in PBS was put into a 96-well plate in the presence of fibrin clots at $37^{\circ} \mathrm{C}$. The fibrin clots were pre-generated via the thrombin-mediated conversion of fibrinogen to fibrin. ${ }^{35}$ For comparison, $100 \mu \mathrm{L}$ PBS, NK solution, and PS@PDA-Fib were respectively dropped into 96-well plates in the presence of fibrin clots. After mixing for $1 \mathrm{~min}$, these samples were measured with a microplate reader for kinetic absorbance at $630 \mathrm{~nm}$ at $1 \mathrm{~min}$ intervals for $130 \mathrm{~min}$. Fig. 5 shows the effect of the NK-loaded microcapsules on thrombosis. The intensity of the absorbance at $630 \mathrm{~nm}\left(\mathrm{OD}_{630}\right)$ reflects the mass of the fibrin clots and the intensity of the PBS control without fibrin clots was considered as the baseline (curve $e$ ). In the sample of PBS with fibrin clots, the intensity of fibrin clots remains nearly constant within the measurement period, indicating that the fibrin clots in PBS are stable, and no thrombolysis happens. In contrast, the intensity of the fibrin clots with NK decreases sharply and reaches baseline at approximately $70 \mathrm{~min}$, confirming the fibrinolytic activity of pure NK. The intensity of fibrin clots with the NK microcapsules decreases first (curve $c$ ) in an identical way to the decline of fibrin clots with pure NK (curve $d$ ), and then slightly fluctuates from $50 \mathrm{~min}$ to $100 \mathrm{~min}$, finally decreasing again to steady value. These results suggest the sharp dissolution of fibrin clots and the quick diffusion of NK from the microcapsules during the first stage. After a stable stage, the release of NK from the microcapsules is restarted and continues to dissolve the fibrin clots. Thus, the first release is from the non-specific adsorbed NK on the surface of the microcapsules and the second release is the gradual diffusion from the inside of the microcapsules. By contrast, the intensity of fibrin clots with PS@PDA-Fib microparticles remains almost steady (curve $b$ ), proving that microparticles without NK loading have little effect on clot dissolution. Therefore, platelet-inspired microcapsules possess high antithrombotic activity with the release of NK.

The antithrombotic activity of the NK-loaded microcapsules was further confirmed by whole blood clotting. The BCI represents the absorbency ratio of blood solution interacting with the microcapsules to the absorbency of the fresh blood solution. In principle, a more considerable BCI means a better anticoagulant property. ${ }^{36}$ Fig. 6 shows the BCI changes of PDA-Fib microcapsules and NK-loaded microcapsules with time. Diluted blood was used as a control. Clearly, there is no difference in the BCI values within $120 \mathrm{~min}$ among the diluted blood, PDA-Fib and NK-loaded microcapsules. This indicates that no clotting happens within $120 \mathrm{~min}$ for all the samples $(\geq 90 \%)$. After 180 min incubation, the BCI values of diluted blood and PDA-Fib microcapsules are below $60 \%$, while that of the NK-loaded microcapsules is still higher than $90 \%$. The

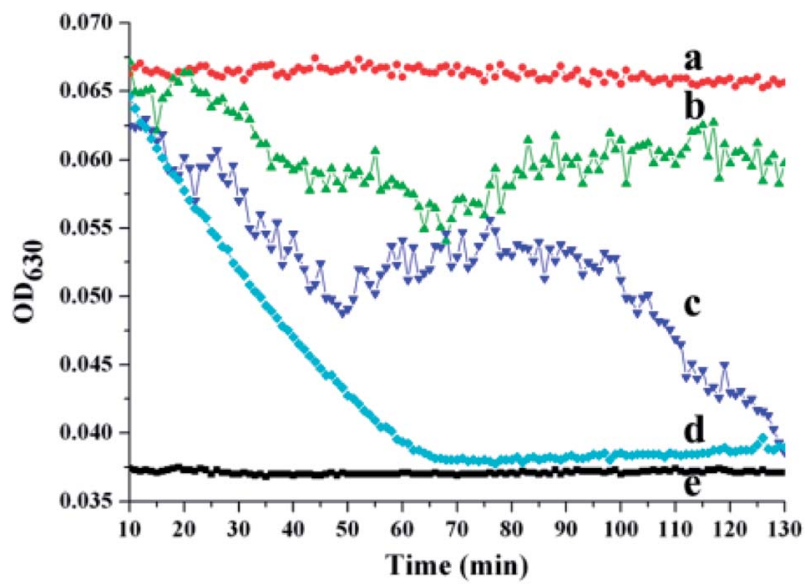

Fig. 5 The antithrombotic activity of (a) PBS with fibrin clots; (b) PDAFib microcapsules with fibrin clots; (c) NK-loaded microcapsules with fibrin clots; (d) NK pure solution with fibrin clots; and (e) PBS as a control. Absorbance at $630 \mathrm{~nm}\left(O D_{630}\right)$ reflects the mass of the fibrin clots. 

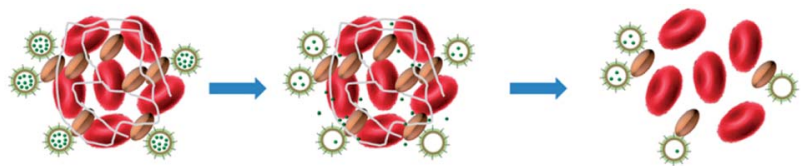

Targeting thrombin

Drug delivery

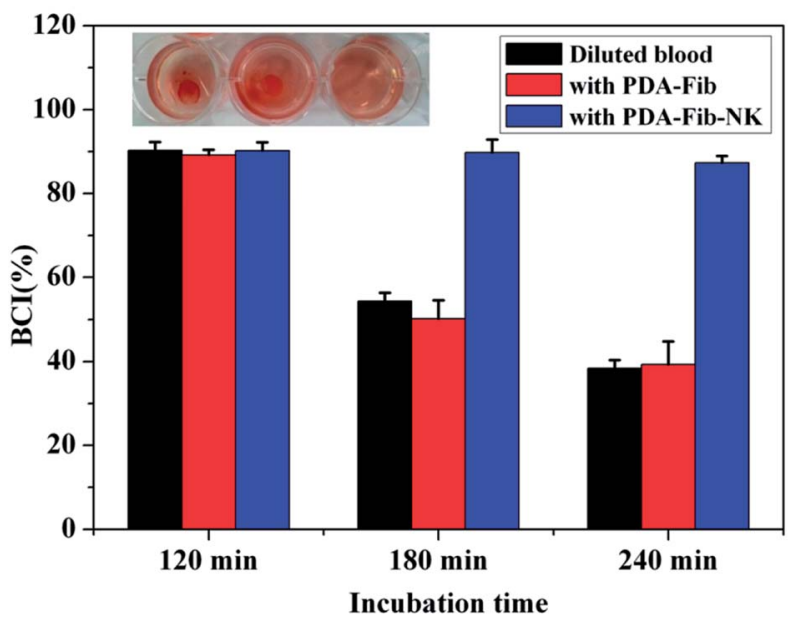

Fig. 6 Blood clotting time indexes (BCls) of diluted blood, fresh blood with PDA-Fib microparticles and fresh blood with PDA-Fib-NK microparticles.

sample containing the NK-loaded microcapsules exhibits no clotting, but the diluted blood and PDA-Fib microcapsule samples become coagulant. These results are consistent with the fibrinolytic activity. The antithrombotic activity of the NKloaded microcapsules is evident within $180 \mathrm{~min}$, even though the release of NK goes down to a stable level within $130 \mathrm{~min}$ (Fig. 5). This is because the activity of NK can be maintained for $3 \mathrm{~h}$ at least. ${ }^{37}$ Astonishingly, no clotting is generated in the NKloaded microcapsule sample within $240 \mathrm{~min}$ (inset photograph), and the BCI value is maintained above $80 \%$. Therefore, the BCI values provide direct evidence of the high antithrombotic effect of the NK-loaded microcapsules. Blood clots are mainly composed of platelets, red blood cells and fibrins formed from Fib during proteolysis by thrombin..$^{38}$ Meanwhile, the formed fibrins can be hydrolyzed by plasmin. NK exhibits plasminogen activator activity and is more sensitive to the cleavage of cross-linked fibrins by directly digesting fibrin through limited proteolysis. ${ }^{39}$ Thus, the mechanism of plateletinspired microcapsules used for targeted antithrombotic drug delivery and release is proposed. Firstly, NK-loaded microcapsules target the activated platelets where the clot forms, and then interact with the platelets in the clot with a high affinity. Secondly, the NK molecules from outside and inside of the microcapsules are released gradually and cleave the fibrins in the clots. Lastly, the high local concentration of NK facilitates fibrinolysis and clot dissolution. The mechanism is schematically described in the top of Fig. 6.

\section{Conclusions}

A facile and versatile method was used to fabricate NK-loaded platelet-inspired microcapsules for targeted antithrombotic drug delivery. This method used PS as a template and PDA as a spacer to immobilize Fib and provide high hydrophilicity. After etching the PS core, a hollow microcapsule was obtained to load NK and perform fibrinolysis in PBS and antithrombosis in blood. The strategy was based on Fib-bound microcapsules that were loaded with NK, which had a high affinity for targeting activated platelets through the interactions between Fib and glycoproteins on the surface of platelets in the blood clots. In this way, the loaded microcapsules exhibited plasminogen activity to induce clot dissolution. The NK-loaded microcapsules with a high loading amount can be regulated to release NK slowly at the site of thrombi. The loading amount of NK was approximately $0.05 \mathrm{mg} \mathrm{g}^{-1}$ at $37^{\circ} \mathrm{C}$, and the loading efficiency was nearly $75 \%$ based on the initial concentration of NK in PBS. The fibrinolytic activity and BCI values proved that the NKloaded microcapsules possess high antithrombotic activity.

This strategy for targeted antithrombotic drug delivery, which lowers the demand dose and minimizes side effects while maximizing drug efficacy, provides a potential treatment for life-threatening diseases caused by vascular obstruction. PDA could be substituted with other biocompatible and biodegradable polymers, such as polyethylene glycol and poly(lactide-coglycolide). NK can be replaced with other antithrombotic agents as well. Thus, our work provides new insight into controlling drug release in targeted antithrombosis treatment with low side effects and high efficiency.

\section{Conflicts of interest}

There are no conflicts to declare.

\section{Acknowledgements}

The authors acknowledge the financial support of the National Natural Science Foundation of China (Projects No. 51503073 and 31500778) and foundation of Huaian Science and Technology Project (HAG201601), Natural Science Foundation of Jiangsu Province (BK20151291).

\section{References}

1 E. Rowin, B. J. Maron, P. Abt, M. Kiernan, A. Vest, F. Costantino, M. Maron and D. DeNofrio, J. Am. Coll. Cardiol., 2018, 71, 681.

2 N. Korin, M. Kanapathipillai, B. D. Matthews, M. Crescente, A. Brill, T. Mammoto, K. Ghosh, S. Jurek, S. A. Bencherif and D. Bhatta, Science, 2012, 337, 738-742.

3 N. Doshi, J. N. Orje, B. Molins, J. W. Smith, S. Mitragotri and Z. M. Ruggeri, Adv. Mater., 2012, 24, 3864-3869.

4 A. Sowińska, E. Czarnowska, M. Tarnowski, J. Witkowska and T. Wierzchoń, Appl. Surf. Sci., 2018, 436, 382-390.

5 E. J. Dunn, H. Philippou, R. A. Ariëns and P. J. Grant, Diabetologia, 2006, 49, 1071-1080.

6 C. F. Greineder, M. D. Howard, R. Carnemolla, D. B. Cines and V. R. Muzykantov, Blood, 2013, 122, 1565-1575.

7 Z. M. Ruggeri, Nat. Med., 2002, 8, 1227-1234. 
8 E. I. Tucker, U. M. Marzec, M. A. Berny, S. Hurst, S. Bunting, O. J. T. Mccarty, A. Gruber and S. R. Hanson, Sci. Transl. Med., 2010, 2, 37ra45.

9 C. Li, W. Ye, J. Jin, X. Xu, J. Liu and J. Yin, J. Mater. Chem. B, 2015, 3, 3922-3926.

10 M. Fujita, K. Hong, Y. Ito, R. Fujii, K. Kariya and S. Nishimuro, Biol. Pharm. Bull., 1995, 18, 1387-1391.

11 H. Chen, L. Yuan, W. Song, Z. Wu and D. Li, Prog. Polym. Sci., 2008, 33, 1059-1087.

12 L. Brass, American Society of Hematology.education Program, Hematology, 2010, 2010, 387-396.

13 W. Ye, Q. Shi, S. C. Wong, J. Hou, X. Xu and J. Yin, Biomater. Sci., 2014, 2, 1186-1194.

14 C. L. Modery-Pawlowski, L. L. Tian, V. Pan, K. R. Mccrae, S. Mitragotri and A. S. Gupta, Biomaterials, 2013, 34, 526541.

15 U. D. S. Sekhon and A. Sen Gupta, ACS Biomater. Sci. Eng., 2018, 4, 1176-1192.

16 C. L. Pawlowski, W. Li, M. Sun, K. Ravichandran, D. Hickman, C. Kos, G. Kaur and A. Sen Gupta, Biomaterials, 2017, 128, 94-108.

17 T. Saxer, A. Zumbuehl and B. Müller, Cardiovasc. Res., 2013, 99, 328-333.

18 F. Gentile, C. Chiappini, D. Fine, R. C. Bhavane, M. S. Peluccio, M. M. Cheng, X. Liu, M. Ferrari and P. Decuzzi, J. Biomech., 2008, 41, 2312-2318.

19 S. M. Marinakos, J. P. Novak, L. C. B. Iii, A. B. House, E. M. Edeki, J. C. F. And and D. L. Feldheim, J. Am. Chem. Soc., 1999, 121, 8518-8522.

20 J. Liebscher, R. Mrówczyński, H. A. Scheidt, C. Filip, N. D. Hădade, R. Turcu, A. Bende and S. Beck, Langmuir, 2013, 29, 10539-10548.

21 H. Lee, S. M. Dellatore, W. M. Miller and P. B. Messersmith, Science, 2007, 318, 426-430.

22 W. Wang, Y. Jiang, S. Wen, L. Liu and L. Zhang, J. Colloid Interface Sci., 2012, 368, 241-249.
23 M. Kim, K. H. Song and J. Doh, Colloids Surf., B, 2013, 112, 134-138.

24 D. R. Jun, S. K. Moon and S. W. Choi, Colloids Surf., B, 2014, 121, 395-399.

25 W. Ye, Q. Shi, J. Hou, J. Gao, C. Li, J. Jin, H. Shi and J. Yin, Appl. Surf. Sci., 2015, 351, 236-242.

26 J. Samal, D. B. Hoban, C. Naughton, R. Concannon, E. Dowd and A. Pandit, Nanomedicine, 2015, 10, 765-783.

27 T. Liao, F. Yuan, C. Shi, C.-X. He and Z. Li, RSC Adv., 2016, 6, 103463-103470.

28 M. Vaithilingam, S. D. Chandrasekaran, S. Gupta, D. Paul, P. Sahu, J. N. Selvaraj and V. Babu, Natl. Acad. Sci. Lett., 2016, 39, 263-267.

29 W. Ye, Q. Shi, J. Hou, J. Jin, Q. Fan, S. C. Wong, X. Xu and J. Yin, J. Mater. Chem. B, 2014, 2, 7186-7191.

30 S. Mitragotri and J. Lahann, Adv. Mater., 2012, 24, 37173723.

31 Q. Liu, B. Yu, W. Ye and F. Zhou, Macromol. Biosci., 2011, 11, 1227-1234.

32 L. Ren, X. Wang, H. Wu, B. Shang and J. Wang, J. Mol. Catal. B: Enzym., 2010, 62, 190-196.

33 M. Juenet, R. Aid-Launais, B. Li, A. Berger, J. Aerts, V. Ollivier, A. Nicoletti, D. Letourneur and C. Chauvierre, Biomaterials, 2018, 156, 204-216.

34 Y. Hu, J. Jin, H. Liang, X. Ji, J. Yin and W. Jiang, Langmuir, 2016, 32, 4086-4094.

35 A. S. Wolberg, Blood Rev., 2007, 21, 131-142.

36 Z. Chang, J. Jing, Z. Jie, J. Wei and J. Yin, Colloids Surf., B, 2013, 102, 45-52.

37 M. Fujita, K. Hong, Y. Ito, S. Misawa, N. Takeuchi, K. Kariya and S. Nishimuro, Biol. Pharm. Bull., 1995, 18, 1194-1196.

38 K. F. Standeven, R. A. Ariëns and P. J. Grant, Blood Rev., 2005, 19, 275-288.

39 J. G. Liu, J. M. Xing, R. Shen, C. L. Yang and H. Z. Liu, Biochem. Eng. J., 2004, 21, 273-278. 